\title{
Survival Outcomes of Hemoperfusion and Hemodialysis versus Hemodialysis in Patients with End-Stage Renal Disease: A Systematic Review and Meta-Analysis
}

\author{
Wendi Chenga Yashuang Luo ${ }^{\mathrm{a}}$ Haiyin Wang ${ }^{\mathrm{a}}$ Xiaoxiao Qin ${ }^{\mathrm{a}}$ Xin Liu ${ }^{\mathrm{a}}$ \\ Yuyan $\mathrm{Fu}^{\mathrm{a}}$ Claudio Ronco ${ }^{\mathrm{b}}$ \\ aDepartment of Health Technology Assessment, Shanghai Health Development Research Center (Shanghai Medical \\ Information Center), Shanghai, China; bepartment of Nephrology, Dialysis and Transplantation, International Renal \\ Research Institute (IRRIV), San Bortolo Hospital, Vicenza, Italy
}

\section{Keywords}

Survival outcomes · Hemodialysis · Hemoperfusion .

End-stage renal disease $\cdot$ Meta-analysis

\begin{abstract}
Background: The maintenance treatment of ESRD puts a great burden on individual patients, society, and the country of China as a whole. Hemoperfusion plus hemodialysis (HP + $\mathrm{HD}$ ) in ESRD patients can yield modified outcomes such as prolonged life expectancy and improved dialysis quality and quality of life. This study aimed to systematically analyze the effect of HP + HD on the overall survival (OS) rates of ESRD patients and to provide support for clinical decision-making. Methods: A computerized search was performed in the PubMed, Embase, Cochrane Library, CNKI, WanFang Data and SinoMed databases for relevant original research articles. Studies were included or excluded based on their compliance with predefined selection criteria. Results: Twelve studies were included in the qualitative synthesis and quantitative synthesis (meta-analysis). The meta-analysis showed that the 1-year OS rate (odds ratio [OR]: $3.35,95 \% \mathrm{Cl}: 1.89$, $5.91, p<0.05$ ), 2-year OS rate (OR: $2.88,95 \% \mathrm{Cl}: 1.84,4.53$, $p<0.05)$, and 5 -year OS rate $\left(X^{2}=4.3092, p<0.05\right)$ of patients with ESRD treated with HP + HD were better than those treated with $H D$, but there was no significant difference in
\end{abstract}

karger@karger.com www.karger.com/bpu

Karger ${ }^{\prime \prime} \div$

BOPEN ACCESS
(C) 2021 The Author(s)

Published by S. Karger AG, Basel

This is an Open Access article licensed under the Creative Commons Attribution-NonCommercial-4.0 International License (CC BY-NC) (http://www.karger.com/Services/OpenAccessLicense), applicable to the online version of the article only. Usage and distribution for commercial purposes requires written permission. 3-year OS rate (OR: $1.97,95 \% \mathrm{Cl}: 0.76,5.06, p>0.05)$. Subgroup analysis showed the same outcomes in different study designs (1-year OS rate: randomized controlled trials [RCTs]: OR: 4.38, 95\% Cl: 1.61, 11.88, $p<0.05$; cohort studies: OR: 2.90, 95\% Cl: 1.44, 5.85, $p<0.05$; 2-year OS rate: RCTs: OR: $2.99,95 \% \mathrm{Cl}: 1.84,4.84, p<0.05)$ and different age-groups (1-year OS rate: $45-50$ years: OR: 3.19, 95\% Cl: $1.55,6.57, p<$ 0.05; 55-60 years: OR: $3.37,95 \% \mathrm{Cl}: 1.07,10.61, p<0.05$; 2-year OS rate: $50-55$ years: OR: $2.86,95 \%$ Cl: $1.59,5.16, p<$ 0.05: 60-65 years: OR: $4.41,95 \% \mathrm{Cl}: 1.19,16.30, p<0.05)$. Conclusions: This meta-analysis suggests that the OS rates of ESRD patients treated with HP + HD were better than those of patients treated with HD. A speculative hypothesis for why this is the case may be that HP + HD can achieve the complementary elimination of metabolites, effectively preventing and treating complications caused by long-term dialysis and prolonging life expectancy. Therefore, HP + HD should be widely used in ESRD patients.

(C) 2021 The Author(s)

Published by S. Karger AG, Basel

\section{Introduction}

CKD is a progressive disease that leads to ESRD, which imposes a substantial public health burden [1]. Globally, the incidence of CKD increased by $89 \%$ to $21,328,972$, the
Correspondence to:

Haiyin Wang,why0522@126.com 
prevalence increased by $87 \%$ to $275,929,799$, death due to CKD increased by $98 \%$ to $1,186,561$, and DALYs increased by $62 \%$ to $35,032,384$ from 1990 to 2016 . The CKD incidence rate per 100,000 population increased from 214.63 (uncertainty interval [UI] = 194.13-234.72) in 1990 to 288.53 (UI $=258.38-319.24$ ) in 2016 [2]. For people aged 60 years and older, several causes, including ischemic heart disease, CKD, and diabetes, ranked among the leading causes of DALYs in 2015 and caused more DALYs than in 2005 [3]. The prevalence of end-stage kidney disease could rise sharply over the next few decades, driven by population aging and an increasing prevalence of diabetes and hypertension [4].

The high cost of maintenance treatment for ESRD puts a great burden on individual patients, society, and the country of China as a whole [5]. The demand for the treatment of ESRD in China is accelerating, which will lead to a rapid increase in the economic burden of ESRD [6]. ESRD is a refractory disease that seriously damages the ability of patients to work. Studies in the USA show that patients with ESRD, who account for fewer than $0.1 \%$ of the total number of patients, consume $1 \%$ of the country's medical resources, similar to those in other Western developed countries [7]. The main treatment methods of ESRD include kidney transplantation, hemodialysis (HD) and peritoneal dialysis. For various reasons, dialysis is the main treatment for ESRD in addition to kidney transplantation $[8,9]$. In recent years, some studies have confirmed that hemoperfusion (HP) combined with HD in the treatment of ESRD patients can achieve the complementary elimination of metabolites, effectively prevent and treat complications caused by long-term dialysis, prolong life expectancy, and improve dialysis quality and patient quality of life. However, there are no systematic reviews or meta-analyses of HP combined with HD on the improvement of the overall survival (OS) rates of ESRD patients. This study aims to systematically analyze whether HP combined with HD improves the OS rates of ESRD patients and to provide support for clinical decision-making.

\section{Methods}

\section{Literature Search Strategy}

A computerized search was performed in the PubMed, Embase, The Cochrane Library, CNKI, WanFang Data, and SinoMed databases (from 2010 to October 2020, totaling 130 months) for original research articles using the following $\mathrm{MeSH}$ terms and freetext keywords ("chronic kidney disease" OR "CKD" OR "end stage renal disease" OR "ESRD" OR "end stage kidney disease" OR "ESKD") AND ("hemodialysis" OR "HD" OR "hemoperfusion"
OR "HP" OR "maintenance hemodialysis" OR "MHD") AND ("survival rate" OR "overall survival" OR "mortality" OR "Progression-Free Survival"), which were used individually and in various combinations with AND or OR to obtain all randomized controlled trials (RCTs) and observational studies about survival outcomes of HP + HD in the ESRD dialysis population. References cited by review articles were also hand-searched to identify additional studies. Articles in both the Chinese and English languages were included.

\section{Inclusion and Exclusion Criteria}

The following predefined inclusion criteria were used to select the eligible studies: (1) study design: RCT or cohort study; (2) population: end-stage kidney disease patients administered hemodialysis in a hospital or nursing home, aged 18 or above, with no restrictions on sex, race or nationality; (3) intervention: HP technique with or without blood purification techniques such as HD, hemofiltration (HF), hemodiafiltration (HDF), etc.; (4) comparison intervention: HD, HF or HDF instead of HP; and (5) outcome measures: OS, progression-free survival, all-cause mortality, cardiovascular mortality, etc.

Studies were excluded from the review if they met the following criteria: (1) repeated publications; (2) review papers, conference abstracts, theses, news articles, and non-peer-reviewed articles; (3) HP was not discussed, or only HP was involved; (4) the relevant survival outcome indicators were not clear, or the original data were not provided; and (5) the patients were children.

\section{Study Selection and Data Extraction}

To assess eligibility, the data and trial quality information were extracted from the articles selected for inclusion independently by 2 investigators. The extracted data were imported into a standardized Excel file and checked by an independent third investigator. Any disagreements were resolved by discussion and consensus. For each eligible study, general information (first author, year of publication, study design, and sample size), baseline demographic and clinical characteristics of the participants (age, sex, and duration of dialysis), interventions/comparison interventions (HP combined with $\mathrm{HD} / \mathrm{MHD} / \mathrm{HF} / \mathrm{HDF}$ and $\mathrm{HD} / \mathrm{MHD} / \mathrm{HF} / \mathrm{HDF}$ treatment), outcome measurement data (OS, progression-free survival, all-cause mortality, and cardiovascular mortality), and literature quality measurements were recorded.

\section{Literature Quality Assessment}

For RCTs, 2 reviewers evaluated the risk of bias of studies according to the Cochrane risk-of-bias tool. The following 6 items were assessed (1) random sequence generation, (2) allocation concealment, (3) blinding, (4) incomplete outcome data, (5) selective outcome reporting, and (6) any other bias (e.g., insufficient rationale and study design).

For observational studies, quality assessment was based on the Newcastle-Ottawa Scale (NOS) with the following items (1) representativeness of the exposed cohort was truly representative, (2) selectin of the nonexposed cohort (drawn from the same community), (3) ascertainment of exposure, (4) demonstration of the outcome of interest was not present at the start, (5a) comparability of the cohorts in age, (5b) comparability of the cohorts in other factor(s), (6) quality of outcome assessment, (7) follow-up time (long enough for outcomes to occur), and (8) complete accounting of all cohorts. All studies were rated on each indicator ( 1 point for 


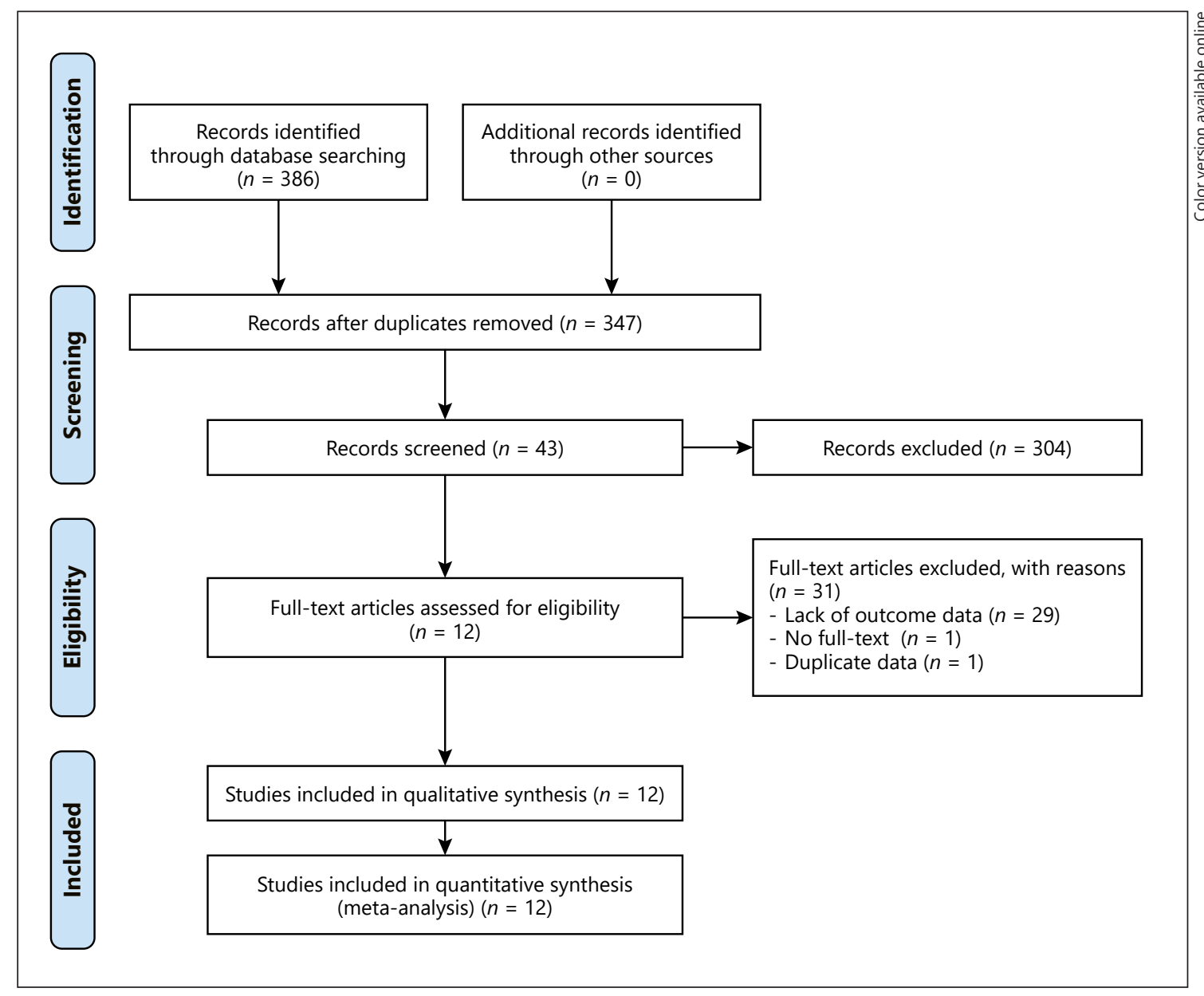

Fig. 1. Flow chart of included studies. The databases retrieved the following numbers of studies: CNKI $(n=25)$, WanFang Data $(n=307)$, SinoMed $(n=1)$, PubMed $(n=17)$, Embase $(n=9)$, Cochrane Library $(n=13)$, and Web of Science $(n=14)$.

"yes" and 0 points for "no") for a total score between 0 and 9 . Scores $\geq 4$ points indicate good quality; scores $<4$ points suggest poor quality. Disagreements were discussed, and third parties were consulted to reach agreement if necessary.

\section{Statistical Analysis}

All data were combined using Review Manager (RevMan, version 5.3.0) (http://ims.cochrane.org/revman). The characteristics of all included studies were tabulated, and datasets were established using Microsoft Excel software according to the data extraction criteria. The Cochran $Q$ test and the $I^{2}$ statistic were used to assess heterogeneity among studies. Heterogeneity was considered present if the $p$ value was $<0.05$ or $I^{2}$ was $>50 \%$. Studies with an $I^{2}$ $\geq 50 \%$ were considered to have heterogeneity. Fixed-effect models by the Peto Mantel-Haenszel method were used when there was no heterogeneity, whereas random-effect models by the DerSimonian-Laird method were applied when there was obvious heterogeneity. $p<0.05$ denoted a statistically significant difference.

For all included studies, odds ratios (ORs) with their 95\% CIs are presented for dichotomous outcomes and displayed in forest plots. The potential publication bias was investigated by the visual inspection of funnel plots for meta-analyses carried out on $>3$ studies. A subgroup analysis was performed based on different study designs and age-groups. Sensitivity analyses were conducted by changing the effect models.

\section{Results}

\section{Search Results}

A total of 386 potentially relevant publications were initially retrieved, and 347 studies remained after removing duplicated articles, of which 304 were excluded for irrelevance after reading their titles and abstracts. Fortythree potentially relevant studies were chosen for full-text analysis, but 29 were excluded because of a lack of key data for analysis, 1 was excluded due to no full-text information, and 1 was excluded for duplicate data. Finally, 12 

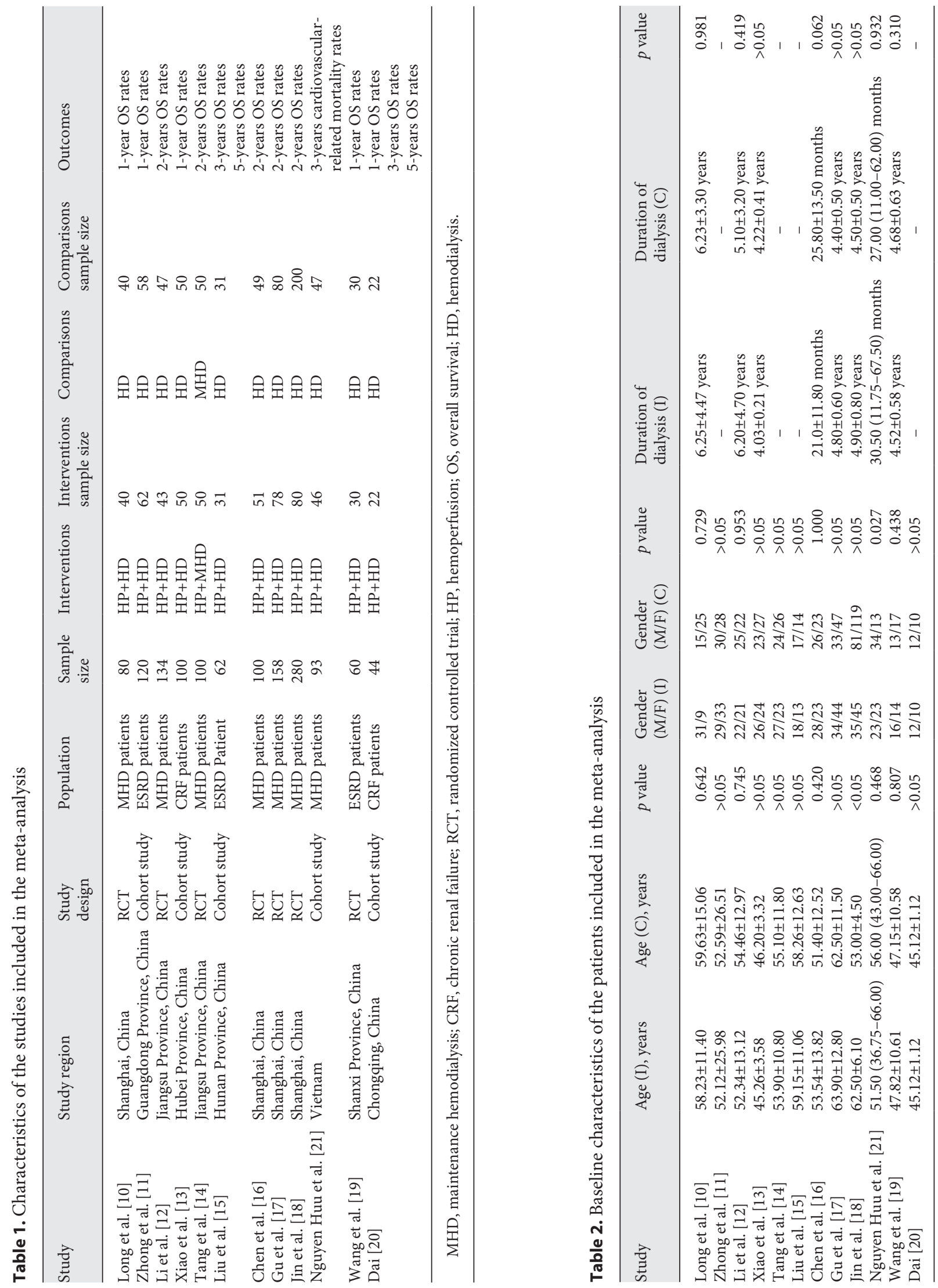
Table 3. Quality assessment of the RCTs included in the meta-analysis

\begin{tabular}{llllll}
\hline Study & $\begin{array}{l}\text { Random } \\
\text { sequence generation }\end{array}$ & $\begin{array}{l}\text { Allocation } \\
\text { concealment }\end{array}$ & Blinding & $\begin{array}{l}\text { Incomplete } \\
\text { outcome data }\end{array}$ & $\begin{array}{l}\text { Selective } \\
\text { outcome reporting }\end{array}$ \\
\hline Long et al. [10] & Low risk & Unclear & Unclear & Low risk & Low risk \\
Gu et al. [17] & Unclear & Unclear & Unclear & Low risk & $\begin{array}{l}\text { Low risk } \\
\text { Low risk }\end{array}$ \\
Jin et al. [18] & Unclear & Unclear & Unclear & Low risk & Unclear \\
Li et al. [12] & Low risk & Unclear & Unclear & Low risk & Low risk \\
Tang et al. [14] & Unclear & Unclear & Unclear & Low risk & Low risk \\
Chen et al. [16] & Low risk & Unclear & Unclear & Low risk & Low risk \\
Wang et al. [19] & Low risk & Unclear & Unclear & Low risk & Low risk \\
\hline
\end{tabular}

RCT, randomized controlled trial.

Table 4. Quality assessment of the cohort studies included in the meta-analysis

\begin{tabular}{|c|c|c|c|c|c|c|c|c|c|}
\hline Zhong et al. [11] & 1 & 1 & 1 & 1 & 2 & 1 & 0 & 1 & 8 \\
\hline Xiao et al. [13] & 1 & 1 & 1 & 1 & 2 & 1 & 0 & 1 & 8 \\
\hline Liu et al. [15] & 1 & 1 & 1 & 1 & 2 & 1 & 1 & 1 & 9 \\
\hline
\end{tabular}

NOS, Newcastle-Ottawa Scale.

studies were included, 9 published in Chinese, and 3 in English [10-21]. The literature screening process and results are shown in Figure 1.

\section{Study Characteristics}

A total of 12 studies were eligible for the final metaanalysis, including 7 RCTs and 5 cohort studies. The main characteristics of the 12 included studies, published between 2011 and 2020, are listed in Table 1. The patients were mainly from China, largely eliminating regional confounding. The sample size of the 12 studies ranged from 44 to 280 , with a total of 1,287 patients, 583 in the intervention group (HP + HD) and 704 in the comparison group (HD). Five studies reported 1-year OS rate, 6 studies reported 2-year OS rate, 2 studies reported 3-year OS rate, 1 study reported 5-year OS rate, and 1 study reported the 3 -year cardiovascular-related mortality rate.

The basic characteristics of the patients included in the study, such as age, sex, and duration of dialysis, are shown in Table 2. The average age of the patients enrolled in the study was 53.78 in the HP + HD group and 53.45 in the HD group. The sex distribution was 301 males and 282 females in the HP + HD group and 333 males and $371 \mathrm{fe}-$ males in the HD group. There was no significant difference in age or sex between the HP + HD group and the HD group in 11 of the studies. The average duration of dialysis was 4.37 years in the HP + HD group and 4.19 years in the HD group. There was no significant difference in the duration of dialysis between the HP + HD group and the HD group in 8 studies.

\section{Literature Quality Assessment Results}

The risk of bias of the included RCTs was evaluated according to the Cochrane risk-of-bias tool for randomized trials (Table 3). Observational studies, were assessed for quality by the NOS (Table 4 ). The NOS score ranged from 8 to 9 , suggesting good quality of the 5 observational studies. 


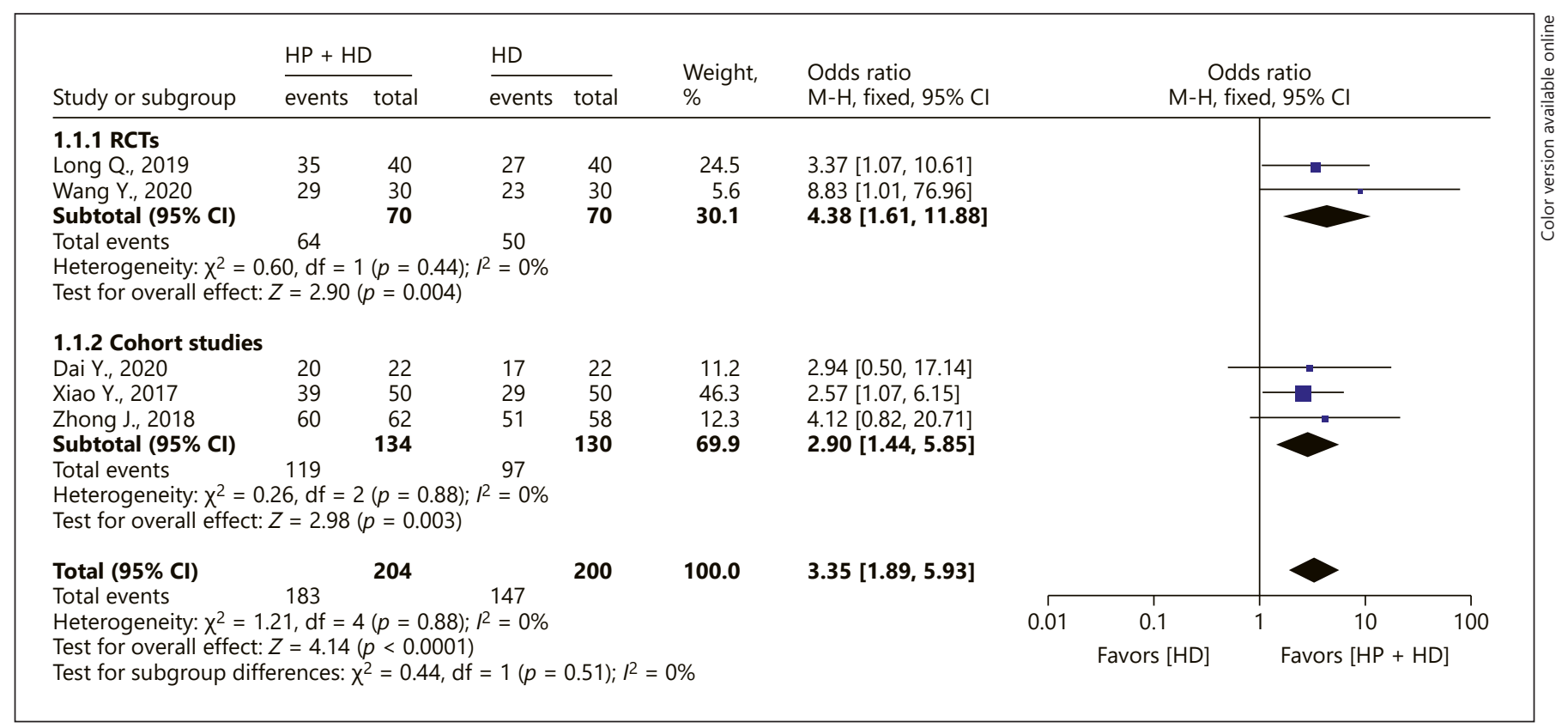

Fig. 2. A forest plot of the meta-analysis of different study design subgroups on the 1-year OS rate in the HP + HD intervention group and the HD control group. HP, hemoperfusion; OS, overall survival; HD, hemodialysis.

\section{Meta-Analysis of Outcome Measures}

One-Year OS Rate in Different Study Design Groups

Five studies reported the 1-year OS rate of patients with ESRD treated with HP + HD. The fixed-effect model of the meta-analysis showed that the 1-year OS rate of patients with ESRD treated with HP + HD was better than that of patients treated with $\mathrm{HD}, \mathrm{OR}=3.35,95 \% \mathrm{CI}(1.89$, 5.93), $p<0.05$, and no heterogeneity was observed $\left(I^{2}=\right.$ $0 \%)$. For the included RCT studies, the fixed-effect model of the meta-analysis showed that the 1-year OS rate of patients with ESRD treated with HP + HD was better than that of patients treated with $\mathrm{HD}, \mathrm{OR}=4.38,95 \% \mathrm{CI}(1.61$, $11.88), p<0.05$. The aggregated results of the included cohort studies showed that the 1-year OS rat of patients with ESRD treated by HP + HD was better than that of patients treated by $\mathrm{HD}, \mathrm{OR}=2.90,95 \% \mathrm{CI}(1.44,5.85)$, $p<0.05$, and no heterogeneity was observed $\left(I^{2}=0 \%\right)$ (Fig. 2).

\section{One-Year OS Rate in Different Age-Groups}

Age-group subgroup analysis was performed for 1 -year OS rate. The 45- to 50-year-old and 55- to 60-yearold patients had increased 1-year OS rate in the fixed-effect model $(45-50$ years old: $\mathrm{OR}=3.19,95 \%$ CI $[1.55$, 6.57], $p<0.05 ; 55-60$ years old: $\mathrm{OR}=3.37,95 \% \mathrm{CI}[1.07$,
$10.61], p<0.05)$. There was no significant difference in the 1-year OS rate in the 50-55-year-old age-group between $\mathrm{HP}+\mathrm{HD}$ and $\mathrm{HD}$ alone, $\mathrm{OR}=4.12,95 \% \mathrm{CI}(0.82$, 20.71), $p=0.09$ (Fig. 3).

Two-Year OS Rate in Different Study Design Groups

Six studies reported the 2-year OS rate of patients with ESRD treated with HP + HD. The fixed-effect model of the meta-analysis showed that the 2-year OS rate of patients with ESRD treated with HP + HD was better than that of patients treated with $\mathrm{HD}, \mathrm{OR}=2.88,95 \% \mathrm{CI}(1.84$, $4.53), p<0.05$, and no heterogeneity was observed $\left(I^{2}=\right.$ $0 \%)$. For the included RCT studies, the fixed-effect model of the meta-analysis showed that the 2-year OS rate of patients with ESRD treated with HP + HD was better than that of patients treated with $\mathrm{HD}, \mathrm{OR}=2.99,95 \% \mathrm{CI}(1.84$, $4.84), p<0.05$. The one cohort study that reported 2 -year OS showed that there was no significant difference in 2 -year OS between $\mathrm{HP}+\mathrm{HD}$ and $\mathrm{HD}$ alone $(\mathrm{OR}=2.22$, $95 \%$ CI $[0.63,7.82], p>0.05$ ) (Fig. 4).

\section{Two-Year OS Rate in Different Age-Groups}

Age-group subgroup analysis was performed for the 2 -year OS rate. The 50- to 55-year-old and 60- to 65-yearold patients had increased 2-year OS rate in the fixed-ef- 


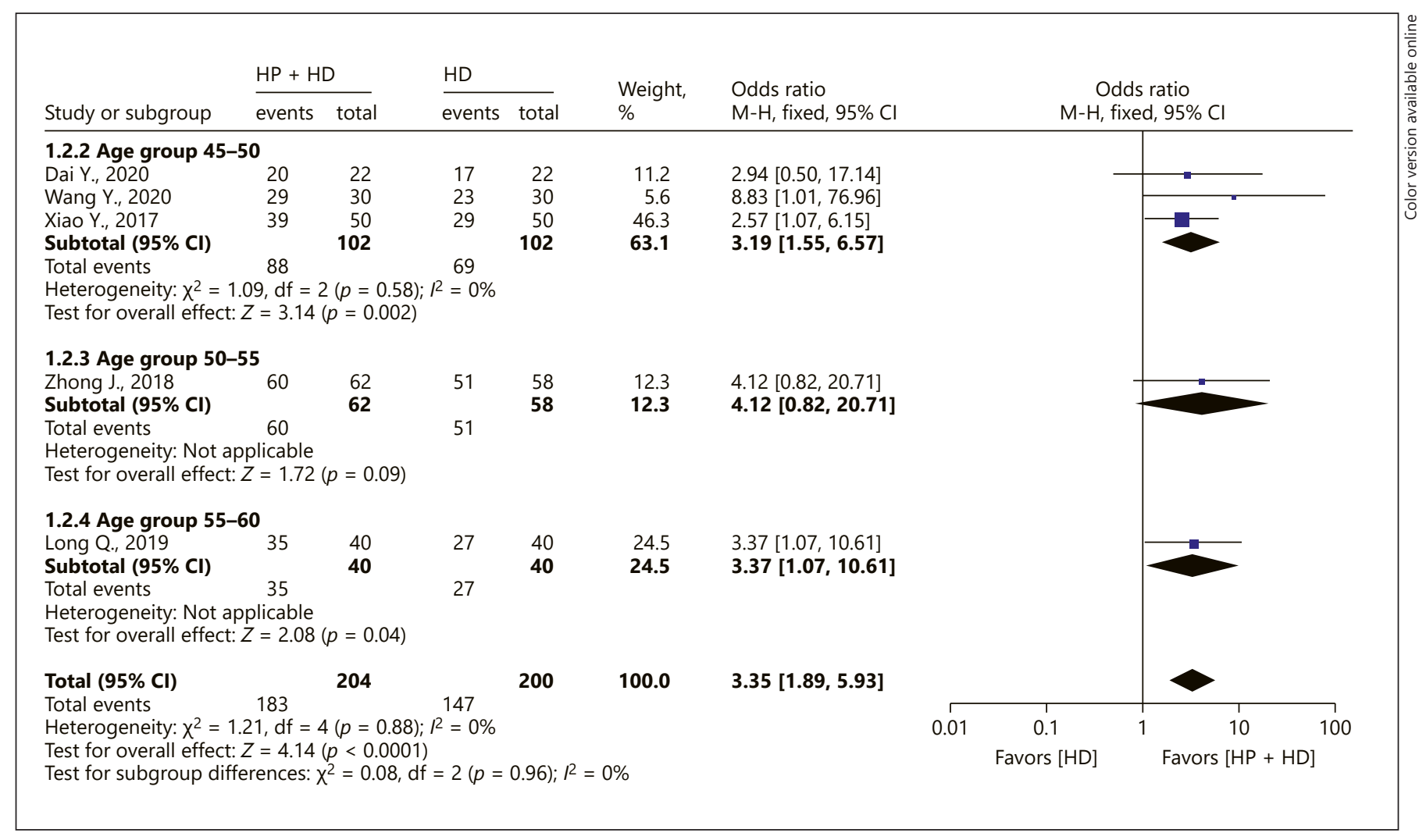

Fig. 3. A forest plot of the meta-analysis of different age subgroups on the 1-year OS rate in the HP + HD intervention group and the HD control group. HP, hemoperfusion; OS, overall survival; HD, hemodialysis.

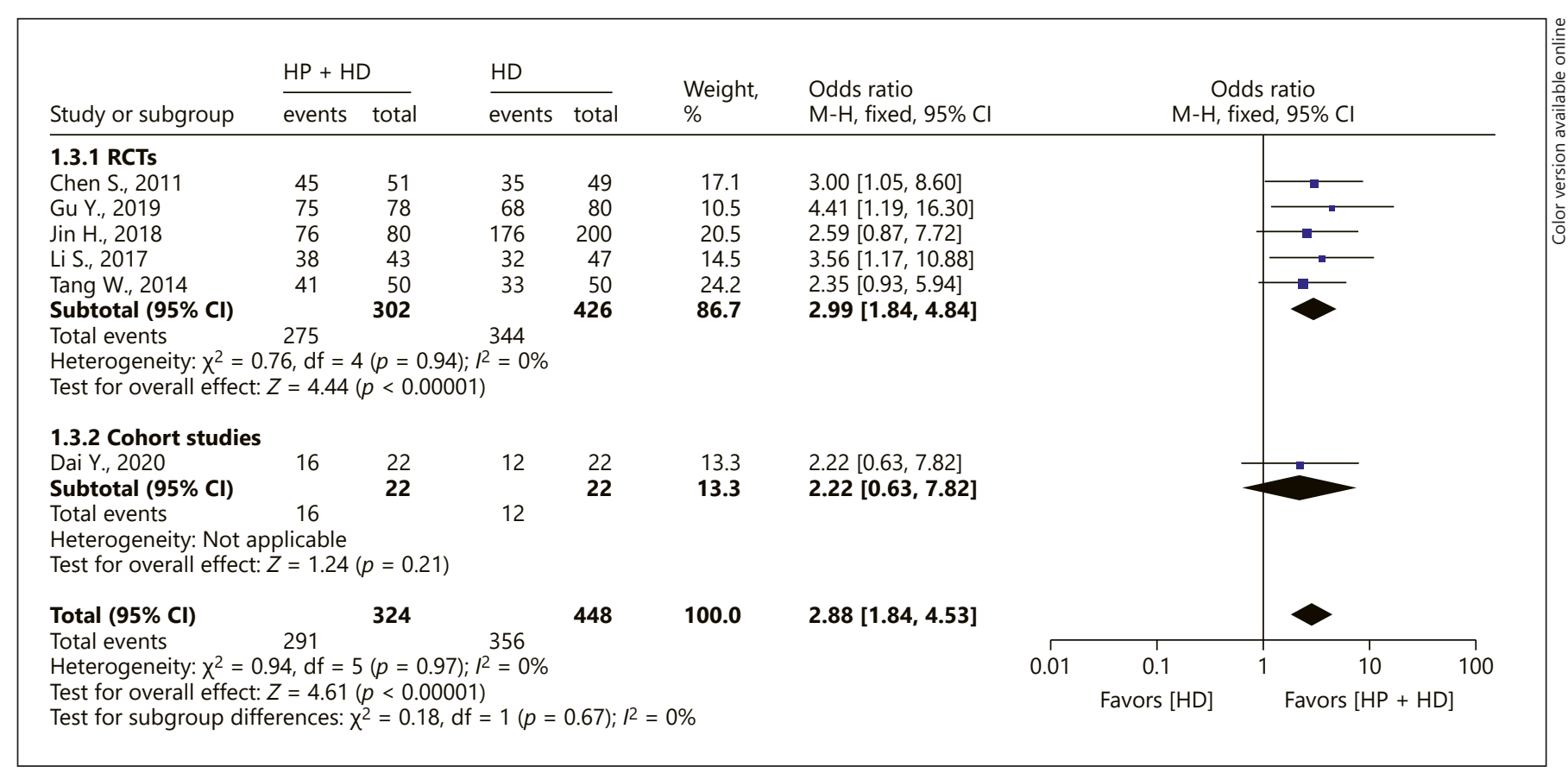

Fig. 4. A forest plot of the meta-analysis of different study-design subgroups on the 2-year OS rate in the HP + HD intervention group and the HD control group. HP, hemoperfusion; OS, overall survival; HD, hemodialysis.

Survival Outcomes of Hemoperfusion and Hemodialysis in ESRD Patients
Blood Purif 2022;51:213-225

DOI: $10.1159 / 000514187$ 


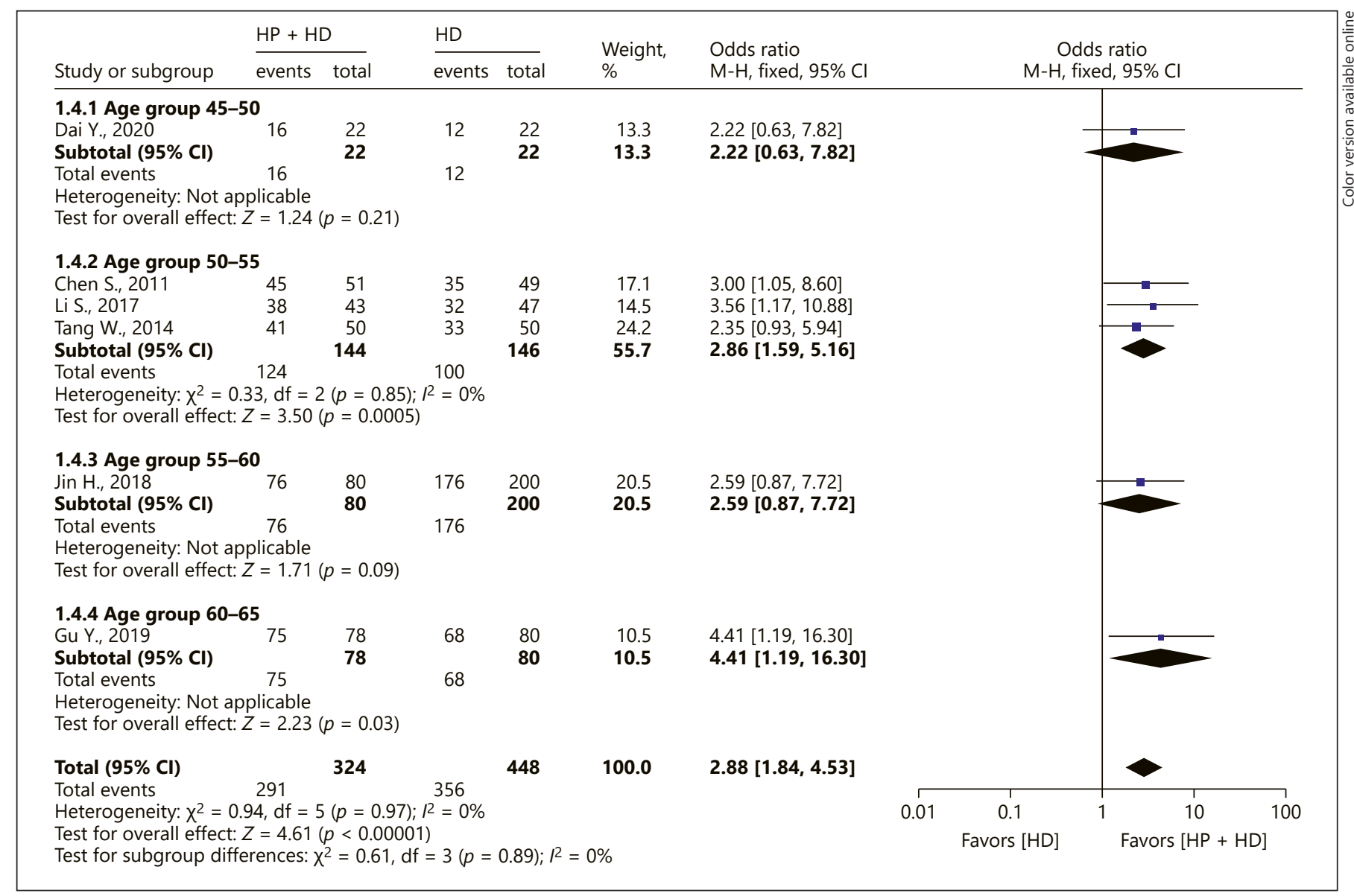

Fig. 5. A forest plot of different age subgroups on the 2-year OS rate in the HP + HD intervention group and the HD control group. HP, hemoperfusion; OS, overall survival; HD, hemodialysis.

fect model $(50-55$ years: $\mathrm{OR}=2.86,95 \%$ CI $[1.59,5.16]$, $p<0.05$; 60-65 years: $\mathrm{OR}=4.41,95 \%$ CI $[1.19,16.30]$, $p<0.05)$. The results of the 1 included study showed that there were no significant differences in the 2-year OS rate of the 45- to 50-year-old or 55- to 60-year-old age-group between HP + HD and HD alone (45-50 years: $\mathrm{OR}=2.22$, $95 \%$ CI $[0.63,7.82], p>0.05 ; 55-60$ years: $\mathrm{OR}=2.59,95 \%$ CI $[0.87,7.72], p>0.05)$ (Fig. 5).

\section{Three-year OS Rate}

Two cohort studies reported the 3-year OS rate of patients with ESRD treated by HP + HD. The fixed-effect model of the meta-analysis showed that there was no significant difference in 3-year OS between ESRD patients treated by $\mathrm{HP}+\mathrm{HD}$ versus $\mathrm{HD}(\mathrm{OR}=1.97,95 \% \mathrm{CI}[0.76$, $5.06], p>0.05)$, and no heterogeneity was observed $\left(I^{2}=\right.$ 0\%) (Fig. 6).
Three-Year Cardiovascular-Related Mortality Rate and 5-Year OS Rate

Only 1 study reported the 3 -year cardiovascular-related mortality rate and 1 study the 5-year OS rate of patients with ESRD treated by HP + HD. Liu [15] showed that the 5 -year OS rate in the HP + HD group was significantly higher than that in the HD group $(p<0.05)$. Nguyen Huu D (2020) showed that the 3-year cardiovascular-related mortality rate in the HP + HD group was significantly lower than that in the HD group $(p<0.05)$.

\section{Potential Publication Bias}

The visual inspection of funnel plots showed no evidence of publication bias among studies reporting 1-year OS rate (Fig. 7). In the funnel plot to detect publication bias among studies reporting 2-year OS rate (Fig. 8), most of the data points were uniformly distributed on both sides of the symmetry axis of the inverted funnel chart 


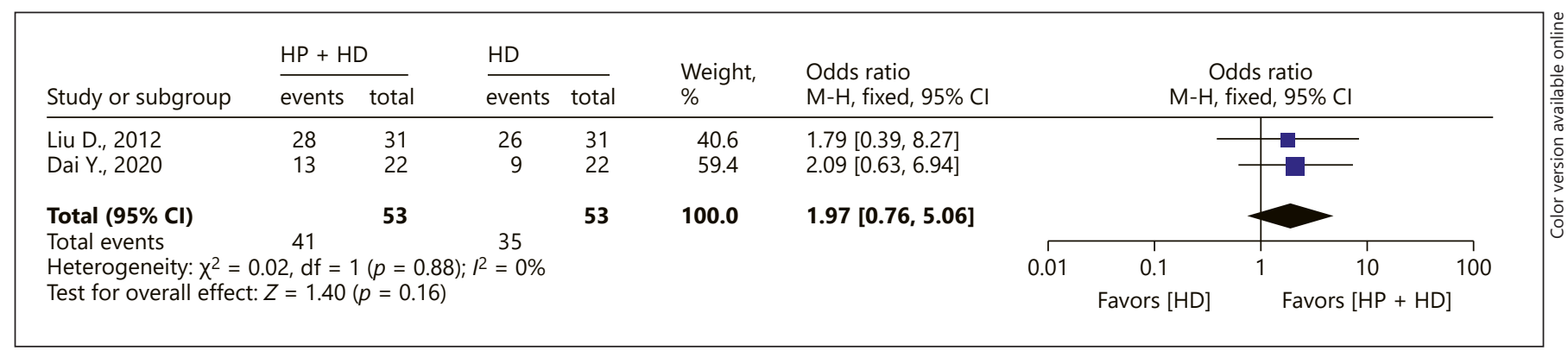

Fig. 6. A forest plot of the meta-analysis of the 3-year OS rate in the HP + HD intervention group and the HD control group. HP, hemoperfusion; OS, overall survival; HD, hemodialysis.

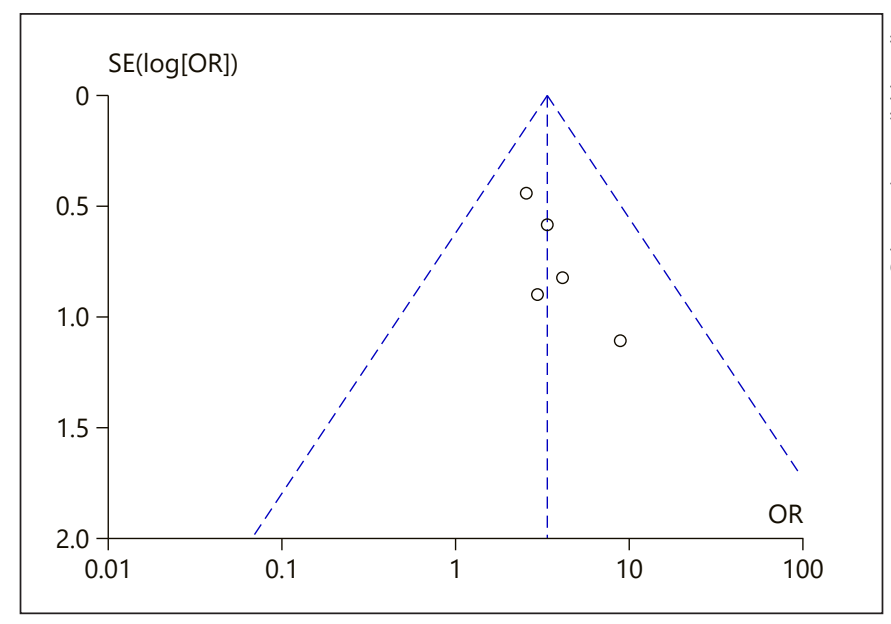

Fig. 7. A funnel plot of 1 -year OS rate in the HP + HD intervention group and the HD control group. HP, hemoperfusion; OS, overall survival; HD, hemodialysis; OR, odds ratio.

and were located in the middle of the inverted funnel chart, suggesting that the research results were not likely to be affected by publication bias.

\section{Sensitivity Analyses}

The sensitivity analyses were conducted by changing the effect models. The 1-year and 2-year OS rate of patients with ESRD treated with HP + HD versus HD alone were still significantly different, and the results showed that the models were robust (Table 5).

\section{Discussion/Conclusion}

This meta-analysis showed that the 1-year OS rate (OR: 3.03 , 95\% CI: $1.61,5.73, p<0.05$ ), 2 -year OS rate (OR: $2.99,95 \%$ CI: $1.84,4.84, p<0.05$ ), and 5 -year OS rate

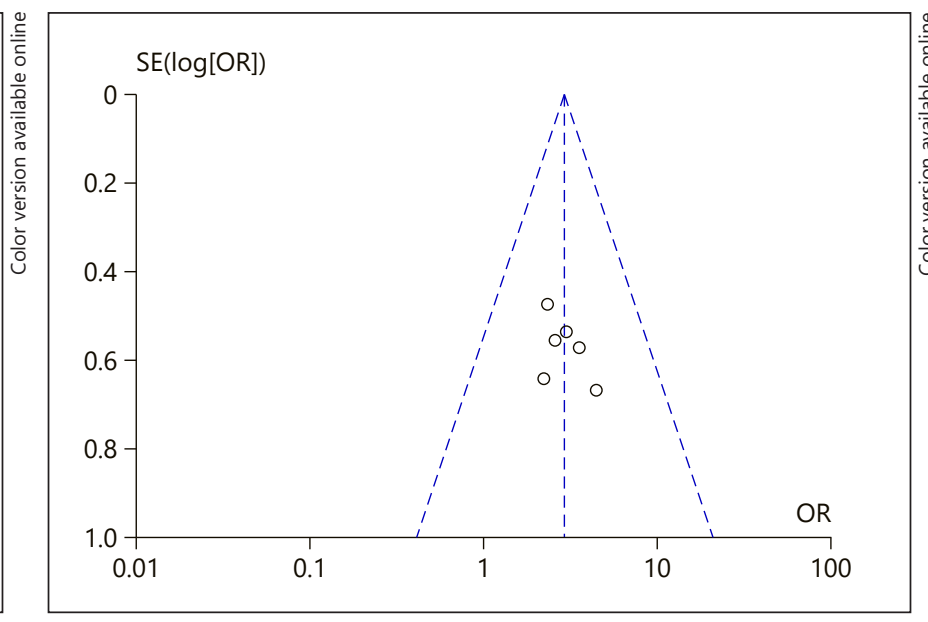

Fig. 8. A funnel plot of 2-year OS rate in the HP $+\mathrm{HD}$ intervention group and the HD control group. HP, hemoperfusion; OS, overall survival; $\mathrm{HD}$, hemodialysis; OR, odds ratio.

$\left(\chi^{2}=4.3092, p<0.05\right)$ of patients with ESRD treated with HP + HD were better than those of ESRD patients treated with HD. Sensitivity analysis showed that the results were robust.

\section{$H P+H D$ to Remove PTH, $\beta 2-M G$, and Other Moderate-Weight and Macromolecular-Weight Uremic Toxins Effectively}

High $\beta 2-M G$ in the body can cause dialysis-related amyloidosis and carpal tunnel syndrome. High PTH can lead to secondary hyperparathyroidism (SHPT). Compared with HD, HP + HD can significantly reduce heart rate, systolic blood pressure, diastolic blood pressure, NYHA grade, and the incidence of hypotension. HP + HD can significantly increase ultrafiltration volume, stabilize hemodynamic indexes more effectively, and have higher dialysis efficiency [11]. The effect of HD + HP on 
Table 5. Sensitivity analyses on OS rates of patients with HP + HD and HD

\begin{tabular}{llllc}
\hline Outcomes & Effect models & OR & $95 \%$ CI & $p$ value \\
\hline 1-year OS rates & Fixed-effect models & 3.35 & $(1.89,5.93)$ & $<0.0001$ \\
& Random-effect models & 3.24 & $(1.82,5.78)$ & $<0.0001$ \\
\hline 2-year OS rates & Fixed-effect models & 2.88 & $(1.84,4.53)$ & $<0.00001$ \\
& Random-effect models & 2.86 & $(1.82,4.49)$ & $<0.00001$ \\
\hline 3-year OS rates & Fixed-effect models & 1.97 & $(0.76,5.06)$ & 0.16 \\
& Random-effect models & 1.97 & $(0.77,5.07)$ & 0.16 \\
\hline
\end{tabular}

OS, overall survival; HP, hemoperfusion; OR, odds ratio; $\mathrm{HD}$, hemodialysis.

reducing the levels of the middle-sized and macromolecular toxins $32-\mathrm{MG}$ and PTH is significantly better than that of $\mathrm{HD}$ alone $[10,12,14-18,21]$. It may be of great benefit to improve SHPT and dialysis-related amyloidosis in MHD patients. After HP + HD treatment, the concentrations of $\mathrm{PTH}, \mathrm{BUN}$, serum $\mathrm{Cr}$ (SCr), serum potassium, and serum phosphorus were lower than those in the HD group, which could effectively improve the clearance of small and medium-sized toxins in patients with chronic renal failure complicated with heart failure, reduce volume load, and improve renal function $[13,20]$.

\section{$H P+H D$ Significantly Improves the State of \\ Microinflammation and Renal Anemia}

A microinflammatory state is common in patients with MHD, which will further lead to atherosclerosis and malnutrition, a state called malnutrition-inflammationatherosclerosis syndrome, which increases the mortality of MHD patients. High-sensitivity C-reactive protein, interleukin-6 (IL-6), and tumor necrosis factor- $\alpha$ (TNF- $\alpha$ ) are most commonly used clinical indicators to reflect the state of microinflammation in patients with MHD. HP + HD can significantly improve the state of microinflammation compared with HD therapy. Compared with HD alone, high-sensitivity $\mathrm{C}$-reactive protein has been significantly reduced in patients treated with $\mathrm{HP}+\mathrm{HD}[10,12$, 14, 16-19], IL-6 has been significantly reduced in patients treated with HP + HD $[10,12,14,16,19]$, TNF- $\alpha$ has been significantly reduced in patients treated with HP + HD $[10,16,19]$, serum leptin has been significantly reduced [16], and inflammatory markers IL-1 and IL-6 have been significantly reduced in patients treated with $\mathrm{HP}+\mathrm{HD}$ [19]. These findings suggest that HP + HD can improve the microinflammatory state of uremic patients. This may be because HP uses neutral macroporous resin adsorption materials, which can effectively adsorb sub- stances with molecular weights of 300-5,000 Da, so it can effectively remove inflammatory mediators such as IL-1, IL-2, IL-6, TNF- $\alpha$, and CRP and block the state of inflammatory reaction in patients. WE speculate that the improvement of anemia in patients with HP + HD may be related to the reduction in microinflammation and oxidative stress in vivo, thus reducing erythropoietin resistance, and may also be related to the improvement of SHPT in patients with MHD.

\section{$H P+H D$ Significantly Improves Left Ventricular}

Hypertrophy, Protects Cardiac Function, and Reduces

\section{Cardiovascular Events}

Left ventricular hypertrophy is one of the most common cardiovascular events in patients with MHD and significantly increases cardiovascular mortality. After HP + HD treatment, the left ventricular mass index of patients decreased significantly, the level of hemoglobin increased, and the level of inflammatory factors decreased. We speculate that the improvement of left ventricular mass index may be related to the improvement of anemia and microinflammation in patients with MHD [10]. The levels of myocardial zymogram CK, LDH, and $\mathrm{HBDH}$ in the HP + HD group were significantly lower than those in the HD group, suggesting that HP + HD treatment can reduce the levels of myocardial enzymes, lighten the burden on the heart more effectively and protect the myocardium [11]. Left ventricular end-diastolic volume, left ventricular end-systolic volume, and left ventricular ejection fraction (LVEF) in the HP + HD group were better than those in the HD group, suggesting that $\mathrm{HP}+\mathrm{HD}$ treatment can improve cardiac function in patients with renal failure [13]. The reason may be that patients with heart failure retain too much water and sodium, and excessive sodium ions and water load can be excreted through blood purification, which can effective- 
ly halt the vicious cycle of heart failure. Blood purification can inhibit the activity of the renin-angiotensin-aldosterone system and reduce the physiology and pathology of heart failure. HP + HD is effective in the treatment of chronic renal failure complicated with heart failure and can improve cardiac function. The incidence of cardio-cerebrovascular events in the HP $+\mathrm{HD}$ group was significantly lower than that in the HD group, indicating that $\mathrm{HP}+\mathrm{HD}$ can effectively reduce the poor prognosis of ESRD patients [19]. This may be because the microinflammatory state is the central link leading to cardiocerebrovascular events in patients with MHD, and $\mathrm{HP}+$ $\mathrm{HD}$ can effectively reduce the expression of inflammatory factors such as IL-1, IL-2, IL-6, TNF- $\alpha$ and CRP, which is helpful to reduce the microinflammatory response mediated by these factors. With the improvement of the microinflammatory state, the incidence and mortality of cardio-cerebrovascular disease also decreased, thus improving the survival and prognosis of patients. In summary, compared with HD alone, HP + HD can effectively improve the level of inflammation and reduce the incidence and mortality of cardio-cerebrovascular disease in patients with ESRD, making it worthy of clinical promotion.

\section{$H P+H D$ Significantly Improves the Quality of Life of \\ Patients}

$\mathrm{HD}+\mathrm{HP}$ can improve the total KDTA score [10, 12], improve the score of 11 dimensions of the KDTA [10], and significantly improve the scores of 6 dimensions (kidney disease, working status, sleep, patient satisfaction, sexual function, and self-health evaluation) of the KDTA [12]. HP + HD has significantly improved the total SF-36 score $[10,12,16]$, the scores of 8 dimensions of SF-36 [10], the scores of 7 dimensions of SF-36 (physical function, physical function, social function, mental health, physical pain, energy status, and overall health expectation) [12], and the scores of 5 dimensions of SF-36 (physical pain, overall health expectation, energy status, emotional function, and mental health) in different studies $[14,16]$. These results suggest that HP + HD has potential advantages in improving the quality of life of patients. In the HP + HD group, skin itching, fatigue, anorexia, sleep quality, body pain, general health, and satisfaction were significantly improved, which may be related to the effective clearance of middle-sized and large molecular toxins such as PTH and $\beta 2-\mathrm{MG}$ by combined therapy, which effectively compensated for the deficiency of simple HD therapy and indicate that it could be widely used in clinical treatment.

Survival Outcomes of Hemoperfusion and Hemodialysis in ESRD Patients
The Benefit of HP $+H D$ in Improving the Survival

Rate of Patients with ESRD

$\mathrm{HP}+\mathrm{HD}$ can better protect the residual renal function (RRF) of patients. A meta-analysis found that HP + HD was associated with increased 1-year, 2-year, and 5-year survival rates in patients with ESRD. RRF is an important determinant of mortality and morbidity in patients undergoing hemodialysis. Different types of dialysis may have different effects on RRF. HP + HD may protect RRF better than $\mathrm{HD}$ alone in patients undergoing maintenance hemodialysis [22].

$\mathrm{HP}+\mathrm{HD}$ can improve microbiota diseases in ESRD patients compared with $\mathrm{HD}$ alone. $\mathrm{HP}+\mathrm{HD}$ can not only enhance the clearance of toxins in the body but also significantly reduce inflammatory factors, which may allow a better bacterial abundance, indicating that different hemodialysis methods have different effects on improving intestinal flora disorders. Therefore, HP + HD can improve the effect of dialysis and play a positive role in improving the imbalance of intestinal flora to improve the prognosis and reduce complications [23].

$\mathrm{HP}+\mathrm{HD}$ can eliminate AGEs to improve the prognosis of patients. Increased AGEs are an independent risk factor for heart disease, and serum levels of toxins such as AGEs rise in the late stages of CKD. The accumulation of these toxins plays an important role in the onset and aggravation of cardiovascular diseases associated with uremia through vascular toxicity. The removal rate of AGEs in the HP + HD group was significantly higher than that in the HD-alone group, which resulted in a better prognosis for patients undergoing maintenance hemodialysis. However, it is necessary to conduct further large-scale studies to follow up on the incidence of and survival rate from CVD in HP + HD [24].

$\mathrm{HP}+\mathrm{HD}$ can improve refractory hypertension. In MHD patients with refractory hypertension, the difficult control of blood pressure is closely related to the excitement of the renin-angiotensin-aldosterone system. Medium- and long-term HP + HD at a certain frequency is a safe, simple, effective, and stable treatment [25].

$\mathrm{HP}+\mathrm{HD}$ can improve peripheral neuropathy. HP + $\mathrm{HD}$ is better than HD in improving ESRD peripheral neuropathy. The occurrence of peripheral neuropathy may be related to the retention of macromolecular toxins in uremia [26].

\section{Limitations}

Although the literature quality assessment results showed good quality of the included studies, only 12 studies were included, and few of them studied the effect of 
$\mathrm{HP}+\mathrm{HD}$ on the survival rate of patients with ESRD, especially in multicenter RCTs. More studies have focused on the clearance of serum $\beta 2-\mathrm{MG}, \mathrm{PTH}$, and other laboratory indicators, RRF indicators, and quality of life indicators. Some other limitations are that only published literature was searched, so there might be publication bias; there were subjective factors in the literature screening and quality assessment; and of the few included studies, those that reported the same outcome indicators were limited.

\section{Conclusions}

The results of the meta-analysis suggest that the OS rates of ESRD patients treated with HP + HD were better than those of patients treated with HD alone because of higher dialysis efficiency, lower levels of middle-sized, and large molecular toxins, improvement of the microinflammatory state, improvement of renal anemia, improvement of left ventricular hypertrophy, better protection of cardiac function, fewer cardiovascular events, improvement of quality of life, and significant improvement of cardiovascular-related mortality. HP + HD should be widely used in clinical treatment.

However, more evidence from RCTs or cohort studies is needed to verify the impact of HP + HD on the longterm survival of patients with ESRD. It is recommended to carry out original studies in this area to provide a reference for clinical practice and decision-making.

\section{Acknowledgements}

The authors would like to thank Professor Claudio Ronco for the English language review.

\section{Statement of Ethics}

The PRISMA (Preferred Reporting Items for Systematic Reviews and Meta-Analyses) standard was used for study selection.

\section{Conflict of Interest Statement}

All the authors declare that they have no conflict of interest.

\section{Funding Sources}

This work was supported by Jafron Biomedical Co., Ltd, China. The funder of the study had no role in study design, data collection, data analysis, data interpretation, or writing of the report.

\section{Author Contributions}

Cheng W. designed this study. Cheng W. and Luo Y. conducted the literature search, data extraction, and subsequent analyses. Cheng W., Qin X., and Liu X. validated and interpreted the results. Cheng W., Luo Y., and Fu Y. drafted the manuscript. Cheng W., Wang H., and C.R. reviewed and edited the manuscript. All the authors read through the manuscript and agreed to the submission of the final version.

\section{References}

1 Moreno Velásquez I, Tribaldos Causadias M, Valdés R, Gómez B, Motta J, Cuero C, et al. End-stage renal disease-financial costs and years of life lost in Panama: a cost-analysis study. BMJ Open. 2019;9(5):e027229.

2 Xie Y, Bowe B, Mokdad AH, Xian H, Yan Y, Li T, et al. Analysis of the global burden of disease study highlights the global, regional, and national trends of chronic kidney disease epidemiology from 1990 to 2016. Kidney Int. 2018;94(3):567-81.

3 Sebaum NJ, Arora M, Barber RM, Bhutta ZA, Brown J, Carter A, et al. GBD 2015 DALYs and HALE collaborators. Global, regional, and national disability-adjusted life-years (DALYs) for 315 diseases and injuries and healthy life expectancy (HALE), 1990-2015: a systematic analysis for the global burden of disease study 2015. Lancet. 2016 Oct; 388(10053):1603-58.
4 Liyanage $\mathrm{T}$, Ninomiya $\mathrm{T}$, Jha $\mathrm{V}$, Neal B, Patrice HM, Okpechi I, et al. Worldwide access to treatment for end-stage kidney disease: a systematic review. Lancet. 2015 May; 385(9981):1975-82.

5 Wang W, Liang H, Lu W. Analysis on treatment burden of end-stage renal disease patients and related policy suggestions. Chin Hea Res. 2018;21(2):121-6.

6 Xiao Y, Sui B, Zhao K. Epidemiological features of the end stage renal disease and the application, cost and payment of dialysis care in China. Chin J Health Pol. 2011;4(5):29-33.

7 Chen Y, Cheng J, Li X. Study on the economic burden of end-stage renal disease dialysis patients. Health Econ Res. 2012;(10):55-6.

8 Cui J, Zhou Z, Tian Q, Lu P, Wang X, Yan Y. Analysis of direct economic burden of dialysis in patients with end stage renal disease in Henan Province. J Med For. 2017;38(10):7-9.
9 Dou W, Zhang W, Wang Y, Lin X, Cai H, Zhou Y, et al. Therapeutic effect of high-flux hemodialysis combined with hemoperfusion on patients with end stage renal disease. J Shanghai Jiaotong Univ. 2015;35(9):1344-7.

10 Long Q, Qin J, Li R, Chang J, Jiang G, Zhu C. Effects of hemodialysis and hemoperfusion combination treatment on maintenance hemodialysis patients. J Shanghai Jiaotong Univ. 2019;39(8):886-92.

11 Zhong J, Huang Z, Li F. Effect of different dialysis methods on the outcome of patients with uremia complicated by heart failure and their effects on myocardial enzyme levels. J.QDU Med. 2018;054(004):407-9.

12 Li S, Liu C, Zhu X. Effect of blood purification on the state of micro inflammation and quality of life in patients with maintenance hemodialysis. J. Qiqihar Univ Med. 2017;38(22): 2636-9. 
13 Xiao Y. Effect of hemodialysis combined with blood perfusion on the treatment effect and long-term survival rate of patients with chronic renal failure and heart failure. J Med Ther Pract. 2017;30(18):2725-6.

14 Tang W, Sun X, Chen R, Zhao X. The observation effect of MHD combined with HP on improving the quality of life and prognosis of maintenance hemodialysis patients. Zhejiang Clin Med J. 2014;12:1958-9.

15 Liu D. Clinical study on combined treatment of hemodialysis and hemodialysis for uremia. Chin Med Guide. 2012;10(30):173-174.

16 Chen S, Lu W, Ji G, Huang H, Wu G, Huang $\mathrm{W}$, et al. Combination of maintenance hemodialysis with hemoperfusion: a safe and effective modality. Chin J Nephrol. 2011;27(1):711.

17 Gu YH, Yang XH, Pan LH, Zhan XL, Guo LL, Jin HM. Additional hemoperfusion is associated with improved overall survival and selfreported sleep disturbance in patients on hemodialysis. Int J Artif Organs. 2019;42(7): $347-53$.
18 Jin HM. Fp462 hemoperfusion improves sleep disturbance and survival in maintain hemodialysis patients. Nephrol Dial Transplant. 2018;33(Suppl 1_1):i191-2.

19 Wang Y, Wang Z, Ma S, Zhang H. Effect of hemoperfusion and hemodialysis on microinflammation and cardiovascular and cerebrovascular events in uremic patients. Chin J Heal C Med. 2020;22(02):173-175.

20 Dai Y. Effect analysis of hemodialysis and hemodialysis series hemoperfusion on survival time of patients. World Latest Medicine Information. 2020;20(12):108-9.

21 Nguyen Huu D, Dao Bui Quy Q, Nguyen Thi Thu H, Phan The C, Nguyen Thi Hong Q, Nguyen Duc L, et al. A Combination of Hemodialysis with Hemoperfusion Helped to Reduce the Cardiovascular-Related Mortality Rate after a 3-Year Follow-Up: A Pilot Study in Vietnam. Blood Purif. 2021;50(1):65-72.

22 Lu W, Ren C, Han X, Yang X, Cao Y, Huang $B$. The protective effect of different dialysis types on residual renal function in patients with maintenance hemodialysis: a systematic review and meta-analysis. Medicine. 2018; 97(37):e12325
$23 \mathrm{He} \mathrm{H}$, Xie Y. Effect of different hemodialysis methods on microbiota in uremic patients. Biomed Res Int. 2020;2020:6739762.

24 Zhang Y, Mei CL, Rong S, Liu YY, Xiao GQ, Shao YH, et al. Effect of the combination of hemodialysis and hemoperfusion on clearing advanced glycation end products: a prospective, randomized, two-stage crossover trial in patients under maintenance hemodialysis. Blood Purif. 2015;40(2):127-32.

$25 \mathrm{Lu} \mathrm{W}$, Xie Y, Huang L, et al. Observation of medium and long term efficacy of hemodialysis combined with hemoperfusion in the treatment of maintenance hemodialysis patients with resistant hypertension. Chin J Blood Purif. 2015;14(05):266-70.

26 Chen X, Chen H, Yang S, et al. The influence of different blood purification profiles on the peripheral neuropathy in end-stage renal disease patients. Chin J Blood Purif. 2012;11(12): 661-3. 